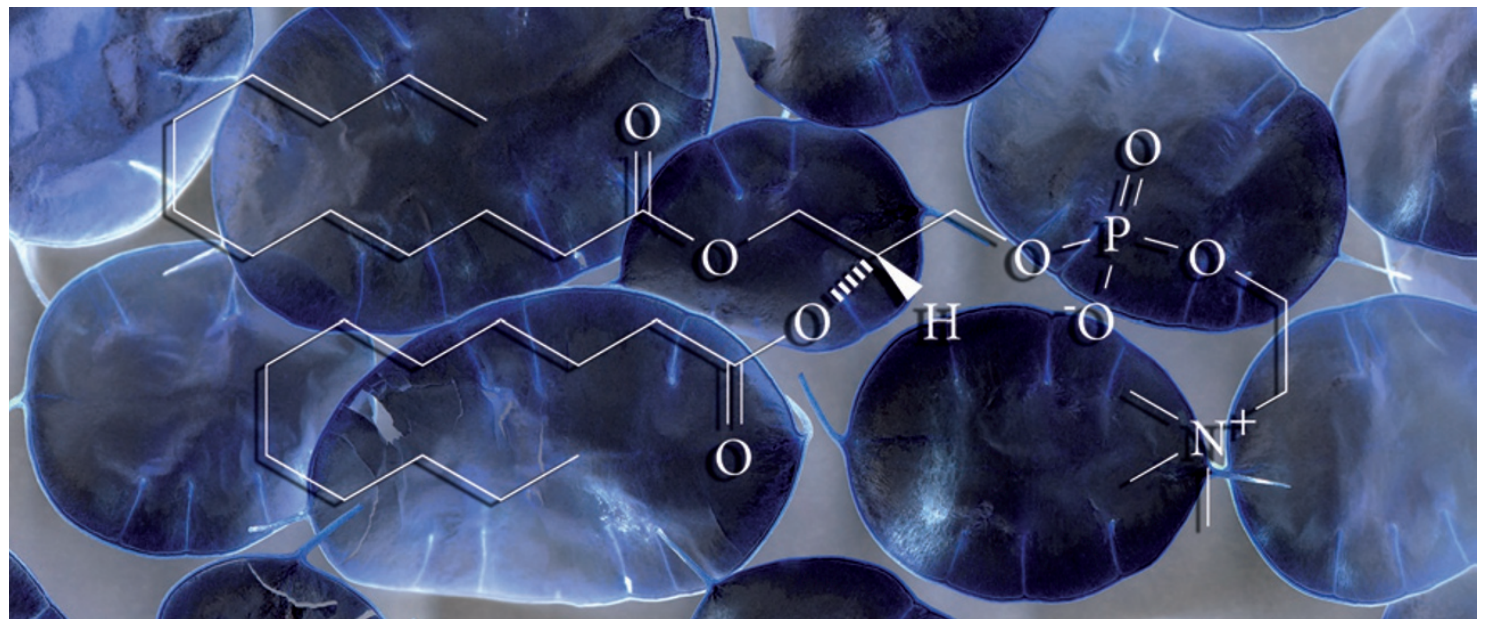

OBESITY AND DIABETES

\title{
An antidiabetic phospholipid
}

An unusual phosphatidylcholine species, dilaroyl phosphatidylcholine (DLPC), reduces fatty liver and improves insulin sensitivity in mice, as described in a recent paper by David Moore and colleagues in Nature. The authors have also discovered that DLPC is a specific agonist of the orphan nuclear receptor LRH1 (also known as NRSA2) and that this receptor is essential for the antidiabetic effects of DLPC.

LRH1 regulates bile acids, which promote the processing of fat and reduce liver steaosis. Because structural studies had suggested that phospholipids might be ligands for LRH1, a phospholipid agonist for this receptor could have useful antidiabetic effects.

To identify potential agonists, the researchers began with in vivo studies using several cell types expressing mouse or human LRH1. By testing the response of LRH1 to various phospholipids, they identified two species - DLPC and diundecanoyl phosphatidylcholine (DUPC) - that activated LRH1. Mutations to LRH1 that are known to block phospholipid binding decreased this effect.

Next, they used a two-hybrid assay to explore to what extent LRH1 interacts with a nuclear receptor coactivator protein, SRC3, in the presence of DLPC, DUPC and other phospholipids. They concluded that only DLPC and DUPC increase this binding. Then, using mass spectrometry, they confirmed that DLPC specifically binds to LRH1. In addition to demonstrating that DLPC and DUPC are specific agonists of LRH1, these in vitro studies showed that DLPC and DUPC do not activate peroxisome proliferator-activated receptor- $\alpha$ (PPAR $\alpha$ ) or PPAR $\gamma-$ nuclear receptors that are known to be activated by other phosphatidylcholine species.

To test the effects of DLPC and DUPC in vivo, the authors administered these phospholipids to $\mathrm{C} 5 \mathrm{BL} / 6$ mice. DLPC and DUPC induced the expression of several LRH1 target proteins that are important for cholesterol and bile acid regulation - cholesterol 7- $\alpha$-monooxygenase (CYP7A1), CYP8B1 and scavenger receptor class $B$ member 1 (SRB1). In addition, DLPC and DUPC repressed SHP - a protein that inhibits the function of LRH1. Mice treated with DUPC or DLPC had elevated liver and serum bile acid levels and reduced levels of liver triglycerides and serum glucose. These effects of DUPC and DLPC were absent in mice lacking the gene for LRH1 in the liver.

Further in vivo studies focused on DLPC, which had shown particularly strong effects. In $\mathrm{db} / \mathrm{db}$ mice fed a high fat diet (a commonly used model of obesity, diabetes and dyslipidaemia), DLPC improved glucose homeostasis. These mice also had lower serum and liver bile acids and less fatty livers. In mice lacking LRH1, however, no beneficial effects were seen.

DLPC also reduced expression of sterol regulatory element-binding protein 1 (SREBP1), a lipogenic transcription factor that is involved in sterol biosynthesis and the regulation of lipids. The authors propose that DLPC may repress SREBP1 through more than one mechanism, creating a beneficial cycle that reduces liver fat deposition and improves insulin sensitivity. This, they say, suggests that DLPC is a promising therapy for metabolic disorders, which they plan to explore in clinical trials.

Samia Burridge

ORIGINAL RESEARCH PAPER Lee, J. M. et al. A nuclear-receptor-dependent phosphatidylcholine pathway with antidiabetic effects. Nature 25 May 2011 (doi:10.1038/nature10111) 